
\title{
25 Research Soure \\ Identification of Core Genes and Pathways in Major Depressive Disorder Applying Bioinformatics
}

\author{
Wenshan Yang \\ Chinese PLA General Hospital https://orcid.org/0000-0002-1666-4261 \\ Hong Yin \\ Chinese PLA General Hospital \\ Yichen Wang \\ Chinese PLA General Hospital \\ Ping Liu \\ Chinese PLA General Hospital \\ Yuan Hu ( $\nabla$ huyuan1980619@126.com ) \\ Chinese PLA General Hospital
}

\section{Research}

Keywords: Major Depressive Disorder, Computational Biology, Critical Pathway

Posted Date: July 17th, 2020

DOI: https://doi.org/10.21203/rs.3.rs-42127/v1

License: (c) (1) This work is licensed under a Creative Commons Attribution 4.0 International License. Read Full License 


\section{Abstract}

Background: Although extensive study efforts on major depressive disorder (MDD), the pathogenesis related to the biological factors are not fully understood and present therapeutic regimen are ineffective in some depressive patients. This study aims to identify key genes and pathways associated with the molecular biological mechanisms of major depressive disorder through bioinformatics analysis in the Gene Expression Omnibus (GEO) public database of the National Center for Biotechnology Information (NCBI) website.

\section{Materials and methods:}

The whole-transcriptome brain expression profile dataset (GSE101521) was obtained from the GEO database. Differentially-expressed genes (DEGs) in normal group (non-psychiatric human) and MDD group (depressive patients) were identified applying Networkanalyst online database. Gene ontology (GO) analysis and Kyoto Encyclopedia of Genes and Genomes (KEGG) pathway analysis were performed to function annotation and enrichment analysis. After that, STRING online database was conducted to protein-protein interaction (PPI) network, and Cytoscape.3.7.2 software was performed to module analysis.

Results: Out of the 41 DEGs identified from normal tissue samples and MDD, 39 were upregulated and 2 were downregulated. GO enrichment analysis discovered that DEGs were primarily involved in inflammatory response, and KEGG pathway analysis suggested that the most chiefly pathway related to MDD were IL-17 signaling pathway, TNF signaling pathway and NOD-like receptor signaling pathway. Six hub genes (IL6, CXCL8, IL1B, FOS, CCL2 and CXCL2) were identified by PPI network and module analysis.

Conclusion: Our current study detected novel markers and targets involved immune system, which are involved in pivotal biological mechanisms related to the pathogenesis of major depression. Looking forward, these findings still need to be validated in future experimental studies.

\section{Background}

Major depression disorder is a serious threat on the physical and mental health of people around the world. The main characteristics of depression are low moods and unhappiness, and depression often accompanied by fatigue, inattention, sleep disturbances and language disorders [1]. The research on the pathogenesis of MDD have seen increasingly rapid advances. According to previous studies, a deficit of monoamines, in particular, serotonin, is one of the important pathogenesis of depression [2], but it has also become increasingly clear that alterations in monoamine systems are not sufficient to explain the full spectra of MDD.

In the search for underlying mechanisms, there was compelling evidence to support the importance of the immune system and synaptic plasticity and the immune system exert a pivotal role on the development of depressive symptoms. It is still extremely crucial to further investigate the molecular mechanisms and 
pathways underlying the etiology and pathogeny of depression to discover novel diagnostic and treatment strategies for depression. Fortunately, with the advances of high-throughput sequencing analyses, noval core genes and pathways have been demonstrated to be correlated with the genesis and progression of depression. For example, Jollant $\mathrm{F}$ et al demonstrated that the serotonergic and nociceptin systems are associated with the activity of depression using peripheral transcriptomic analyses [3]. Nilay et al discovered that Plenty of evidence for IFN-a related molecular biological mechanisms related to depression through whole-blood transcriptomic analyses [4]. Wang et al argued that NF-kappa B signaling pathway were important for onset and development of depression by the analysis of the highthroughput mRNA expression data sets [5]. Carboni et al discovered that growth factor signaling and inflammatory responses imbalanced in both depressive patients and depression symptoms animal models by hippocampal transcriptional [6]. Therefore, investigating hub genes and pathways to illuminate the interactions network among hub genes and pathways are fundamental for further understanding the etiopathogenesis of major depressive disorders.

In our current study, we obtained dataset of Whole-transcriptome brain expression sequences from Gene Expression Omnibus (GEO) public database, and founded many pivotal genes and pathways as biomarkers in major depressive disorders by applying bioinformatics analysis. And the potential hub genes and pathways we discovered can further help us better understanding pathogenesis underlying major depressive disorder.

\section{Materials And Methods}

\section{Whole-transcriptome Data and Identification of DEGs.}

The mRNA expression profile dataset GSE101521 was obtained from the GEO public database (https://www.ncbi.nlm.nih.gov/geo/query/acc.cgi?acc=GSE101521)[7]. Gene expression data information in GSE101521 based on the Illumina MiSeq human platform GPL15520. In this dataset, twenty-nine non-psychiatric human dorsal lateral prefrontal cortex samples were collected from sudden death non-psychiatric individuals, and thirty samples were collected from major depressive disorder (MDD) with/without suicides individuals. The GSE101521 dataset was divided into two groups: normal and MDD groups. The counts data from GSE101521 datasets were chosen for normalization using networkanalyst (https://www.networkanalyst.ca/) website through edgeR. DEGs were identified by networkanalyst website between normal and MDD groups. The cutoff criteria were set as follows: |log2Fold Changel $>1$ and adj-P value $<0.05$. The study design is shown in a flow diagram (Figure 1 ).

\section{Gene Ontology (GO) and KEGG pathway enrichment analysis}

GO enrichment analysis were performed applying the Database for Annotation, Visualization and Integrated Discovery (DAVID), an online gene annotation software (https://DAVID.ncifcrf.gov/)[8, 9]. In order to select the best biological process (BP), cellular components (CC), and molecular function (MF), the most possible functions involved in DEGs were screened. KEGG is a database that integrates genes, compounds, and regulatory networks in known biological processes, and analyzes gene function and 
genome at the system level. In our present study, KEGG pathway analysis were conducted by KOBAS (http:// http://kobas.cbi.pku.edu.cn/kobas3) [10]. The p-value $<0.05$ was considered statistically significant.

\section{Protein-protein interaction network analysis}

The STRING 11.0 online software (https://string-db.org/) [11] and Cytoscape (version: cytpscape.3.7.2) [12] were performed to analyze protein-protein interaction network for DEGs. The STRING 11.0 online database was performed to identify the interactions between DEGs and the Cytoscape software was conducted to visualize the results. The MCODE plugin of Cytoscape was conducted to identify key clusters of PPI network and cytohubba plugin of Cytoscape was performed to identify hub genes. Degree cut-off $=2$, node score cut-off $=0.2, \mathrm{k}$-core $=2$, and max. depth $=100$ were consider as the default parameters criteria, and degree $>10$ was considered as hub gene. Genes in the key clusters were performed functional enrichment and KEGG pathway analysis again.

\section{Results}

\section{Identification of DEGs}

According to the criteria adj-P<0.05 and $|\log 2(\mathrm{FC})|>1,41$ DEGs were identified when comparing the normal and MDD groups. A total of 39 DEGs were upregulated and 2 were downregulated. DEGs were shown in Figure.2 and Table.1.

\section{GO and KEGG enrichment analyses of DEGs.}

GO enrichment analysis was carried out on the 41 DEGs using DAVID online database. In terms of BP, DEGs were primarily involved in inflammatory response, positive regulation of angiogenesis, and cellular response to heat (Figure.3A). In terms of CC, DEGs were most enriched in extracellular space, extracellular region and cytosol (Figure.3B). In terms of MF, DEGs mainly participated in unfolded protein binding and heat shock protein binding (Figure.3C). KEGG enrichment results suggested that DEGs were primarily enriched in IL-17 signaling pathway, Legionellosis, and Measles (Figure.3D). GO enrichment analysis and KEGG pathway suggested that the most associated biological function was the inflammatory response.

\section{PPI network}

In order to dig out the highest depression-associated genes, PPI network analysis was conducted by STRING 11.0 online database, and 41 DEGs were mapped into PPI network containing 28 nodes and 108 edges (Figure.4A). Our PPI network analysis results predicted that the 28 DEGs had effects on the pathogenesis of depression. Three clusters were screened by the MCODE plugin of Cytoscape in the PPI network. And between them, the cluster 1 contained 10 nodes (FOS, SELE, ATF3, CXCL8, TNFAIP3, IL6, IL1B, C5AR1, CCL2, CXCL2) and 37 edges with the highest score (score=8.222) (Figure.4B); the cluster 2 contained 4 nodes and 6 edges (score $=4.000)$ (Figure.4C), the cluster 3 contained 3 nodes and 3 edges (score=3.000) (Figure.4D). 
Function enrichment analysis were conducted again for DEGs of the cluster1, which had the highest MCODE score. For GO enrichment analysis, the DEGs of cluster 1 were mostly enriched in inflammatory response and immune response (Figure.5A). KEGG analysis suggested that the DEGs of cluster 1 were mostly enriched in IL-17 signaling pathway, TNF signaling pathway, and NOD-like receptor signaling pathway. All six hub genes (CCL2, CXCL2, FOS, CXCL8, IL6 and IL1B) were mapped on IL-17 signaling pathway, and five hub genes (CXCL2, CCL2, IL1B, FOS, IL6) were founded to be mapped on the TNF signaling pathway (Figure.5B). Furthermore, five hub genes (CCL2, CXCL2, CXCL8, IL6 and IL1B) were also identified to be associated with NOD-like receptor signaling pathway in cluster 1. Six genes (IL6, CXCL8, IL1B, FOS, CCL2 and CXCL2) were considered as hub genes by the cytohubba plugin of Cytoscape in the cluster1 (degree>10).

\section{Discussion}

In the current study, we screened 41 DEGs and built the hub genes (IL6, CXCL8, IL1B, FOS, CCL2, CXCL2) by PPI network and module analysis, and last KEGG pathway analysis revealed that the most chiefly pathway related to MDD were IL-17 signaling pathway, TNF signaling pathway and NOD-like receptor signaling pathway. This study indented novel markers and targets involved immune system which may participate in pivotal mechanism involved in the pathogenesis of major depressive disorder.

Whole-transcriptome and next generation sequencing have produced numerous data sets including IncRNA, miRNA, and mRNA expression profiles with the development of high throughput technologies. A mass of core new genes and pathways were identified to predict potential pathogenesis of MDD by using bioinformatics analysis. Different from other studies, here, we acquired datasets (GSE101521) of wholetranscriptome expression profile from GEO, and applied bioinformatics analyses to find hub genes and pathways connected to MDD. We submitted the DEGs at the significance level of corrected $p$ value $<0.05$ to the DAVID online database and KOBAS online database for GO enrichment functional and KEGG pathway analysis. The results from DAVID for GO enrichment functional annotation for DEGs and the significantly expressed genes assigned to KEGG pathway analysis suggested that the top significant term was inflammatory response as a key functional signature related to the pathogenesis of depressive patients. The GO enrichment of DEGs was mainly related to biological process including inflammatory response, positive regulation of angiogenesis, cellular response to heat, response to unfolded protein, immune response, cellular response to lipopolysaccharide, etc. Furthermore, we founded that the significantly DEGs mapped to KEGG pathway analysis were IL-17 signaling pathway, TNF signaling pathway, and NOD-like receptor signaling pathway, which were involved in MDD. Some of the results of previous study were accordant to our current research demonstrated that immune and inflammatory response are initially considered to be related to the underlying biological response system which involved in the etiopathogenesis of MDD. [13-15].

Increased inflammatory cytokines and inflammatory activation in the brain exert crucial roles in the etiopathogenesis of MDD [16]. Some omics studies also have published that the IL-17 signaling pathway and TNF signaling pathway were related to the depression process $[17,18]$. TNF-a regulates microglia 
activation through the NF-KB signaling pathway and pro-inflammatory cytokines, and thus plays a crucial part in the neuroinflammation [19]. Activation of NF-KB signaling pathway triggered the cytokines and chemokines by IL-17 signals [20], while inhibiting the function of the IL-17 cytokine family could have a beneficial effect on pathogenic conditions in CNS [21,22]. And NF-KB signaling cascades could also activate NOD-like receptor (NLR) [23], and activation of NLR inflammasome lead to subsequent release of IL-1B by human macrophages [24]. At the same time Combined with the above research evidence and our results, here, we speculated that NF-KB signaling cascades and activation of NOD-like receptor signalingmight induced the unbalance of IL-17 signaling and TNF signaling, and result in a series of immune inflammation response include release of cytokines and chemokine (IL-1B, IL-6, CCL2, CXCL2) which were crucial in pathogenesis of major depressive disorder (Figure.6).

But in fact, the present study has some limitations. The database in this study was performed from postmortem brains, it might not exactly the same with the living tissue, so that whether the targets could be extended to living tissue remains unknow and some of targets might lost. Hence, in future study, more proteomic or RNA-seq, in MDD patients were in plan to exploring the molecular biology mechanisms of major depressive disorder. In addition, hub genes and pathways we founded should be verified by vivo and vitro experiments.

\section{Conclusions}

In conclusion, our present research demonstrated that hub genes (IL6, CXCL8, IL1B, FOS, CCL2, CXCL2), which participate in pivotal mechanism related to the etiopathogenesis of MDD, were activated via IL-17 signaling pathway, TNF signaling pathway and NOD-like receptor signaling pathway, which might demonstrate novel markers and targets enriched immune inflammation pathogenesis of major depressive disorder. However, these findings still need to be validated in future experimental studies.

\section{Abbreviations}

MDD: major depressive disorder; DEGs: differentially expressed genes; GO: Gene ontology; PPI: proteinprotein interaction; IL6: interleukin 6; CXCL8: chemokine (C-X-C motif) ligand 8; IL1B: interleukin 1 beta; FOS: Fos proto-oncogene; CCL2: C-C motif chemokine ligand 2; CXCL2: C-X-C motif chemokine ligand 2; GEO: Gene Expression Omnibus; DAVID: The Database for Annotation, Visualization and Integrated Discovery.

\section{Declarations}

\section{Ethics approval and consent to participate}

Not applicable

\section{Consent for publication}


Consent for publication was obtained from all participants

\section{Availability of data and material}

All data generated or analyzed during this study are included in this article

\section{Competing interests}

The authors declare that there are no conflicts of interest.

\section{Funding}

This work was supported by National Natural Science Foundation of China (N0.81973502).

\section{Author contributions}

Wenshan Yang and Hong Yin contributed to the design of the study and prepared manuscript. Yichen Wang made the data acquisition, Ping Liu provided the several suggestions for manuscript revision. Yuan Hu contributed to study supervision and final approval of the manuscript.

\section{Acknowledgements}

Not applicable

\section{References}

1. Serretti A, et al. Depressive syndrome in major psychoses: a study on 1351 subjects. Psychiatry Res. 2004;127(1-2):85-99.

2. Heim C, et al. The link between childhood trauma and depression: insights from HPA axis studies in humans. Psychoneuroendocrinology. 2008;33(6):693-710.

3. Jollant F, et al., Neural and molecular correlates of psychological pain during major depression, and its link with suicidal ideas. Prog Neuropsychopharmacol Biol Psychiatry, 2020: p. 109909.

4. Hepgul N, et al. Transcriptomics in Interferon-alpha-Treated Patients Identifies Inflammation-, Neuroplasticity- and Oxidative Stress-Related Signatures as Predictors and Correlates of Depression. Neuropsychopharmacology. 2016;41(10):2502-11.

5. Wang $\mathrm{H}$, et al. Identification of diagnostic markers for major depressive disorder by cross-validation of data from whole blood samples. PeerJ. 2019;7:e7171.

6. Carboni L, et al. Cross-species evidence from human and rat brain transcriptome for growth factor signaling pathway dysregulation in major depression. Neuropsychopharmacology. 2018;43(10):2134-45.

7. Pantazatos SP, et al. Whole-transcriptome brain expression and exon-usage profiling in major depression and suicide: evidence for altered glial, endothelial and ATPase activity. Mol Psychiatry. 
2017;22(5):760-73.

8. Huang da W, Sherman BT, Lempicki RA. Systematic and integrative analysis of large gene lists using DAVID bioinformatics resources. Nat Protoc. 2009;4(1):44-57.

9. Huang da W, Sherman BT, Lempicki RA. Bioinformatics enrichment tools: paths toward the comprehensive functional analysis of large gene lists. Nucleic Acids Res. 2009;37(1):1-13.

10. Xie $\mathrm{C}$, et al., KOBAS 2.0: a web server for annotation and identification of enriched pathways and diseases. Nucleic Acids Res, 2011. 39(Web Server issue): p. W316-22.

11. Szklarczyk D, et al. STRING v10: protein-protein interaction networks, integrated over the tree of life. Nucleic Acids Res. 2015;43(Database issue):D447-52.

12. Shannon P, et al. Cytoscape: a software environment for integrated models of biomolecular interaction networks. Genome Res. 2003;13(11):2498-504.

13. Fleshner M, Frank M, Maier SF. Danger Signals and Inflammasomes: Stress-Evoked Sterile Inflammation in Mood Disorders. Neuropsychopharmacology. 2017;42(1):36-45.

14. Kiecolt-Glaser JK, Derry HM, Fagundes CP. Inflammation: depression fans the flames and feasts on the heat. Am J Psychiatry. 2015;172(11):1075-91.

15. Miller $\mathrm{AH}$, Raison $\mathrm{CL}$. The role of inflammation in depression: from evolutionary imperative to modern treatment target. Nat Rev Immunol. 2016;16(1):22-34.

16. Slavich GM, Irwin MR. From stress to inflammation and major depressive disorder: a social signal transduction theory of depression. Psychol Bull. 2014;140(3):774-815.

17. Yi Y, et al. The core genes involved in the promotion of depression in patients with ovarian cancer. Oncol Lett. 2019;18(6):5995-6007.

18. An T, et al. Baduanjin exerts anti-diabetic and anti-depression effects by regulating the expression of mRNA, IncRNA, and circRNA. Chin Med. 2019;14:3.

19. Kong $X$, et al. TNF-alpha regulates microglial activation via the NF-kappaB signaling pathway in systemic lupus erythematosus with depression. Int J Biol Macromol. 2019;125:892-900.

20. Gu C, Wu L, Li X. IL-17 family: cytokines, receptors and signaling. Cytokine. 2013;64(2):477-85.

21. Waisman A, Hauptmann J, Regen T. The role of IL-17 in CNS diseases. Acta Neuropathol. 2015;129(5):625-37.

22. Qian $Y$, et al. The adaptor Act1 is required for interleukin 17-dependent signaling associated with autoimmune and inflammatory disease. Nat Immunol. 2007;8(3):247-56.

23. Choi AJ, Ryter SW. Inflammasomes: molecular regulation and implications for metabolic and cognitive diseases. Mol Cells. 2014;37(6):441-8.

24. Engin A. The Pathogenesis of Obesity-Associated Adipose Tissue Inflammation. Adv Exp Med Biol. 2017;960:221-45.

\section{Tables}


Table 1

The differential expressed genes were identified when comparing the normal and MDD groups.

\begin{tabular}{|c|c|c|c|c|c|}
\hline Ensembl GenelD & Gene Symbol & $\log F C$ & PValue & adj.P.Val & Up/down-regulated \\
\hline ENSG00000173110 & HSPA6 & 5.8737 & 7.23E-16 & $2.30 \mathrm{E}-11$ & up-regulated \\
\hline ENSG00000225217 & HSPA7 & 4.4626 & $9.99 \mathrm{E}-13$ & $1.59 \mathrm{E}-08$ & up-regulated \\
\hline ENSG00000255823 & MTRNR2L8 & -3.5736 & 7.15E-12 & $7.58 \mathrm{E}-08$ & down-regulated \\
\hline ENSG00000149257 & SERPINH1 & 2.8477 & $1.86 \mathrm{E}-11$ & $1.48 \mathrm{E}-07$ & up-regulated \\
\hline ENSG00000169429 & CXCL8 & 3.7158 & 2.09E-10 & $1.33 \mathrm{E}-06$ & up-regulated \\
\hline ENSG00000007908 & SELE & 3.1341 & 7.36E-09 & 2.74E-05 & up-regulated \\
\hline ENSG00000108691 & CCL2 & 2.3278 & 7.73E-09 & $2.74 \mathrm{E}-05$ & up-regulated \\
\hline ENSG00000125538 & IL1B & 2.4031 & $2.37 \mathrm{E}-08$ & $5.79 \mathrm{E}-05$ & up-regulated \\
\hline ENSG00000261441 & AC124068.2 & 2.7239 & $5.78 \mathrm{E}-06$ & 0.007557 & up-regulated \\
\hline ENSG00000136244 & IL6 & 3.0355 & $9.60 \mathrm{E}-06$ & 0.010355 & up-regulated \\
\hline ENSG00000129988 & LBP & 2.378 & 4.43E-05 & 0.033271 & up-regulated \\
\hline ENSG00000162772 & ATF3 & 1.8009 & 7.74E-09 & $2.74 \mathrm{E}-05$ & up-regulated \\
\hline ENSG00000170345 & FOS & 1.7223 & $1.73 \mathrm{E}-08$ & $5.00 \mathrm{E}-05$ & up-regulated \\
\hline ENSG00000118503 & TNFAIP3 & 1.618 & $1.14 \mathrm{E}-07$ & 0.00026 & up-regulated \\
\hline ENSG00000138135 & $\mathrm{CH} 25 \mathrm{H}$ & 1.2212 & $4.45 \mathrm{E}-07$ & 0.000943 & up-regulated \\
\hline ENSG00000132002 & DNAJB1 & 1.269 & 7.87E-07 & 0.001479 & up-regulated \\
\hline ENSG00000204389 & HSPA1A & 1.55 & $7.90 \mathrm{E}-07$ & 0.001479 & up-regulated \\
\hline ENSG00000217527 & RPS16P5 & 1.9163 & $1.04 \mathrm{E}-06$ & 0.00183 & up-regulated \\
\hline ENSG00000204388 & HSPA1B & 1.4886 & $1.12 \mathrm{E}-06$ & 0.001876 & up-regulated \\
\hline ENSG00000198805 & PNP & 1.0734 & $1.76 \mathrm{E}-06$ & 0.002802 & up-regulated \\
\hline ENSG00000166592 & RRAD & 1.5785 & $2.12 \mathrm{E}-06$ & 0.003215 & up-regulated \\
\hline ENSG00000125740 & FOSB & 1.5646 & 4.16E-06 & 0.006012 & up-regulated \\
\hline ENSG00000115008 & IL1A & 1.6225 & $5.33 \mathrm{E}-06$ & 0.007372 & up-regulated \\
\hline ENSG00000137801 & THBS1 & 1.2124 & $5.94 \mathrm{E}-06$ & 0.007557 & up-regulated \\
\hline ENSG00000106211 & HSPB1 & 1.2338 & 7.34E-06 & 0.008704 & up-regulated \\
\hline ENSG00000124762 & CDKN1A & 1.3617 & $8.49 \mathrm{E}-06$ & 0.009652 & up-regulated \\
\hline ENSG00000081041 & CXCL2 & 1.8547 & $1.27 \mathrm{E}-05$ & 0.013026 & up-regulated \\
\hline
\end{tabular}




\begin{tabular}{|llllll|}
\hline Ensembl GenelD & Gene Symbol & logFC & PValue & adj.P.Val & Up/down-regulated \\
\hline ENSG00000105825 & TFPI2 & 1.9105 & $1.80 E-05$ & 0.017307 & up-regulated \\
\hline ENSG00000197405 & C5AR1 & 1.1513 & $2.20 E-05$ & 0.020577 & up-regulated \\
\hline ENSG00000143333 & RGS16 & 1.0112 & $2.47 E-05$ & 0.022019 & up-regulated \\
\hline ENSG00000128016 & ZFP36 & 1.3924 & $2.71 E-05$ & 0.023341 & up-regulated \\
\hline ENSG00000006327 & TNFRSF12A & 1.5202 & $3.15 E-05$ & 0.026388 & up-regulated \\
\hline ENSG00000151929 & BAG3 & 1.3813 & $3.57 E-05$ & 0.029147 & up-regulated \\
\hline ENSG00000146410 & MTFR2 & 1.3841 & $3.84 E-05$ & 0.030542 & up-regulated \\
\hline ENSG00000214814 & FER1L6 & -1.6258 & $4.04 E-05$ & 0.03133 & down-regulated \\
\hline ENSG00000139151 & PLCZ1 & 1.4809 & $4.50 E-05$ & 0.033271 & up-regulated \\
\hline ENSG00000148926 & ADM & 1.3525 & $5.00 E-05$ & 0.034381 & up-regulated \\
\hline ENSG00000146904 & EPHA1 & 1.0071 & $5.14 E-05$ & 0.034381 & up-regulated \\
\hline ENSG00000133874 & RNF122 & 1.2358 & $5.19 E-05$ & 0.034381 & up-regulated \\
\hline ENSG00000109321 & AREG & 1.8551 & $5.83 E-05$ & 0.036681 & up-regulated \\
\hline ENSG00000230650 & AC140479.2 & 1.8274 & $6.89 E-05$ & 0.042138 & up-regulated \\
\hline
\end{tabular}

Figures 


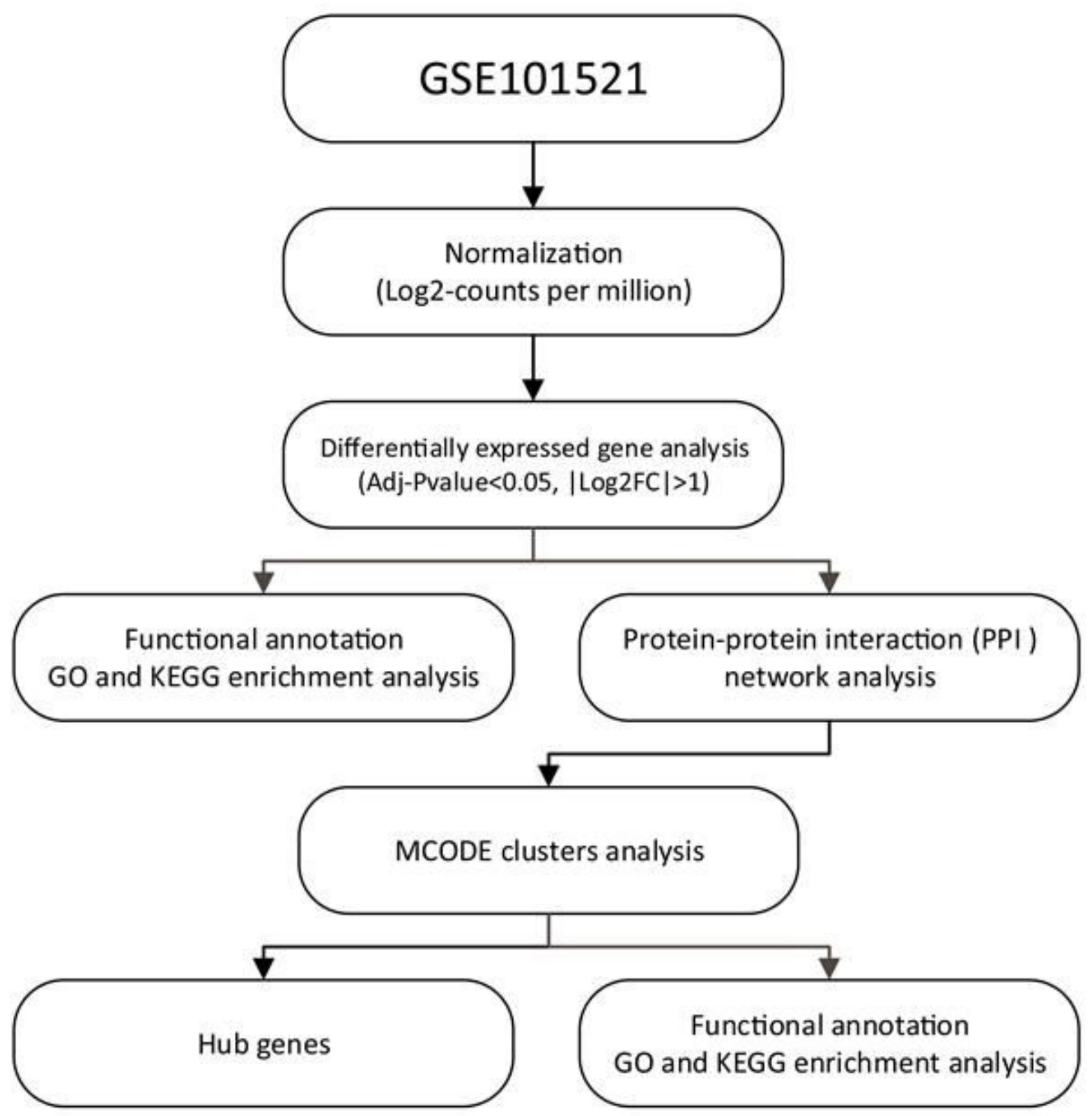

Figure 1

Flow diagram of our present study. 


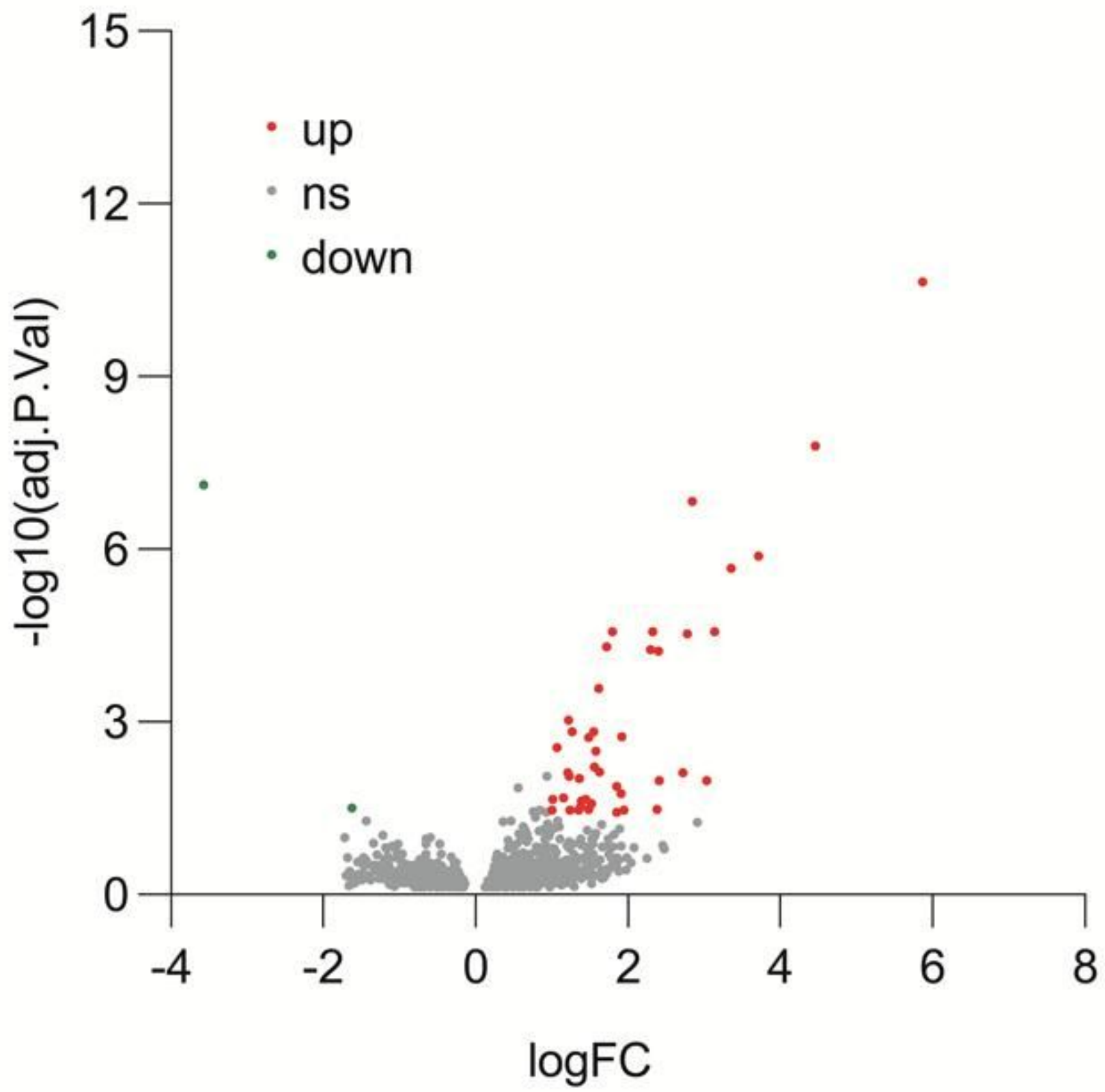

Figure 2

Volcano plot of differential expression genes. Red points as up-regulated DEGs, green plots as downregulated DEGs, and gray plots as genes with no significant difference. 
A The top 10 enriched $\mathrm{GO}$ terms (BP)

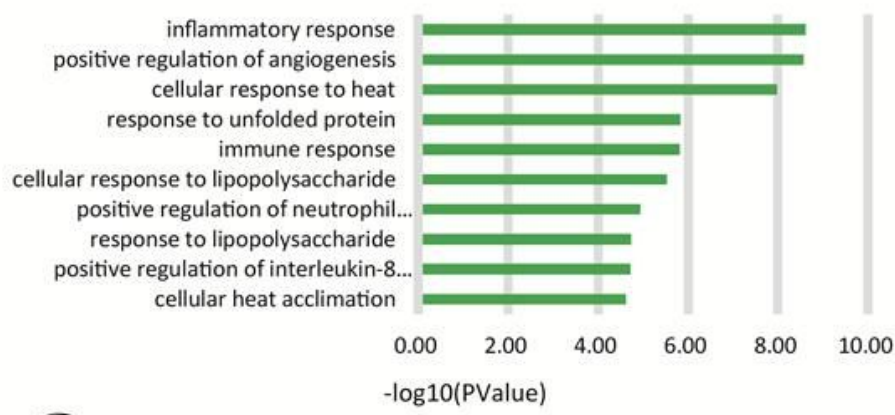

C

\section{The top 10 enriched GO terms (MF)}

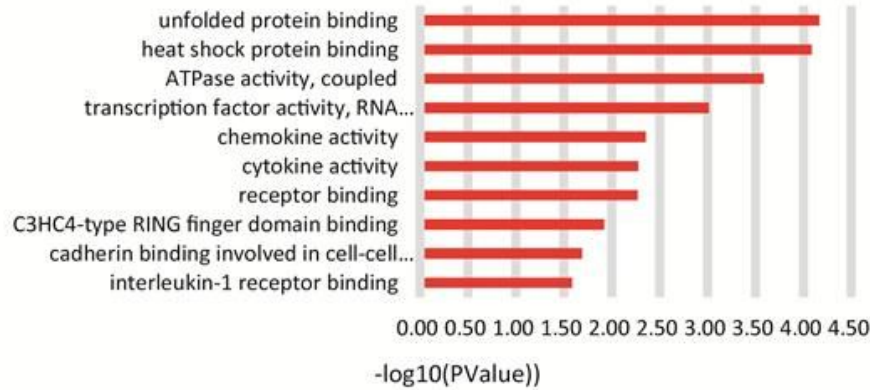

$\mathrm{B}$ The whole enriched GO terms (CC)

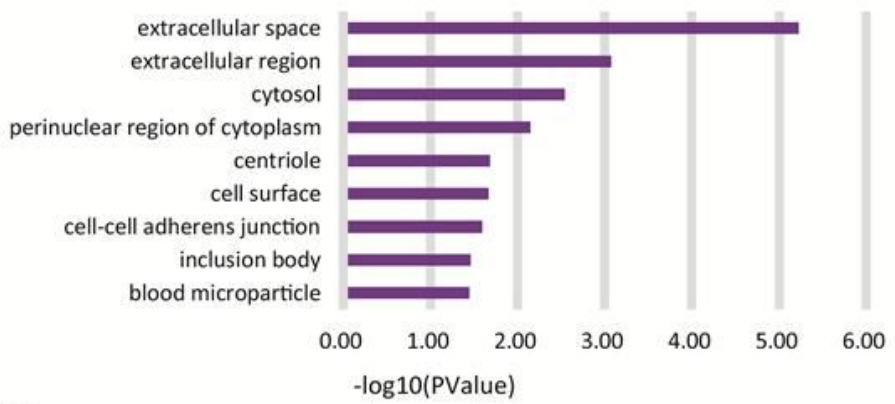

The top 10 enriched KEGG pathway

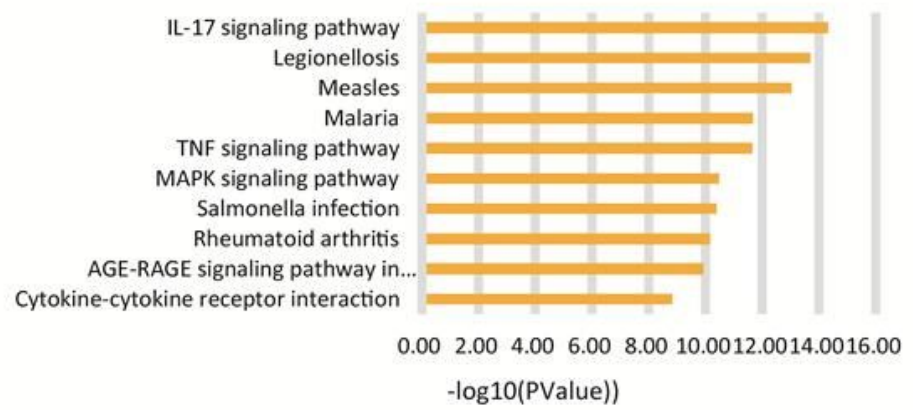

\section{Figure 3}

GO and KEGG enrichment analyses of DEGs; (A) The top 10 enrichen GO terms of DEGs (BP); (B) The top 10 enrichen GO terms of DEGs (CC); (C) The top 10 enrichen GO terms of DEGs (MF); (D) The top 10 enrichen KEGG pathways of DEGs. 

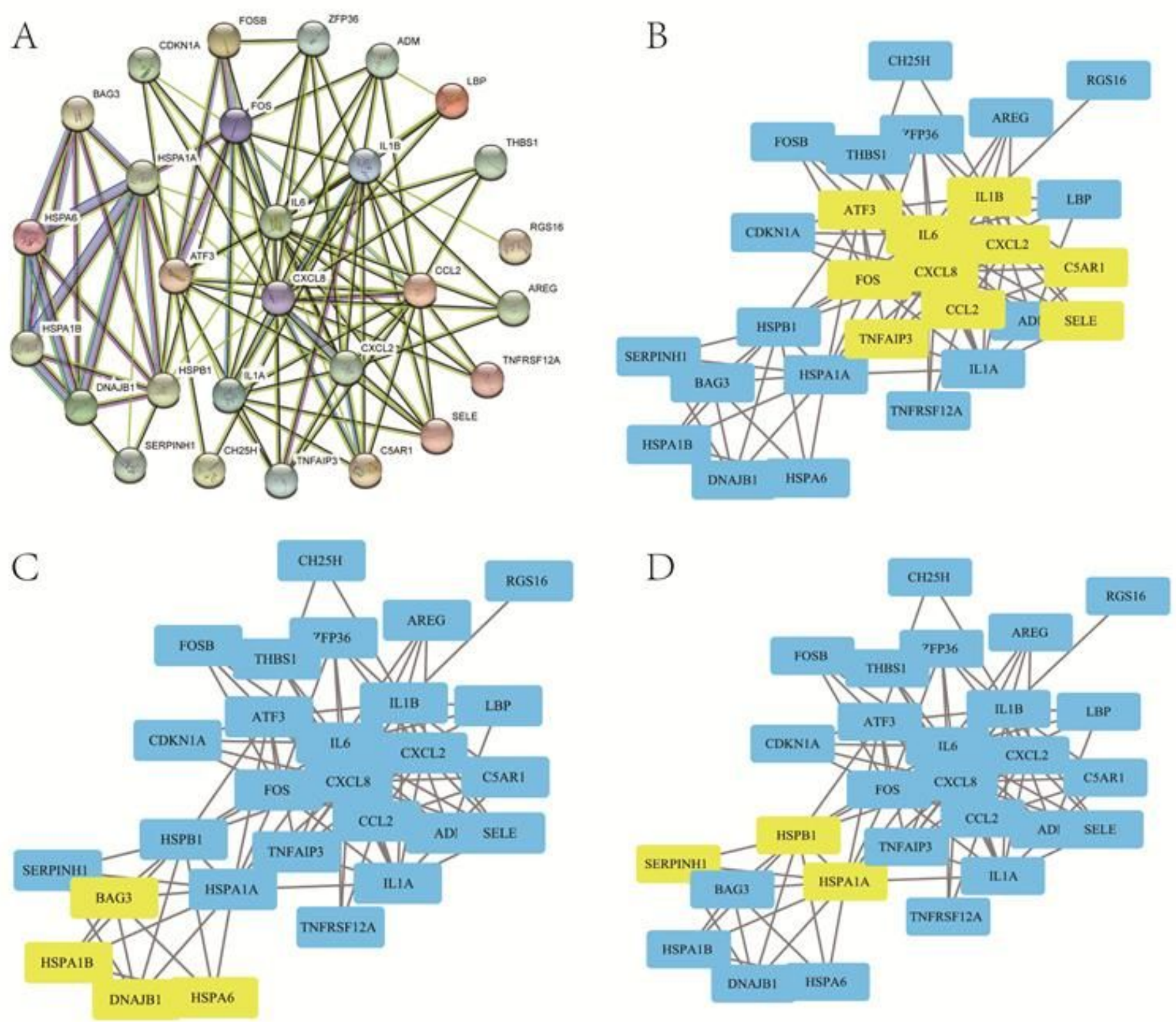

Figure 4

PPI network analysis of DEGs and MCODE clusters analysis; (A)The PPI network. Proteins were represented with color nodes, and interactions were presented with edges. (B) The cluster1 of PPI network; (C) The cluster2 of PPI network; (D) The cluster3 of PPI network. 


\section{The top 10 enriched GO terms (cluster1)}

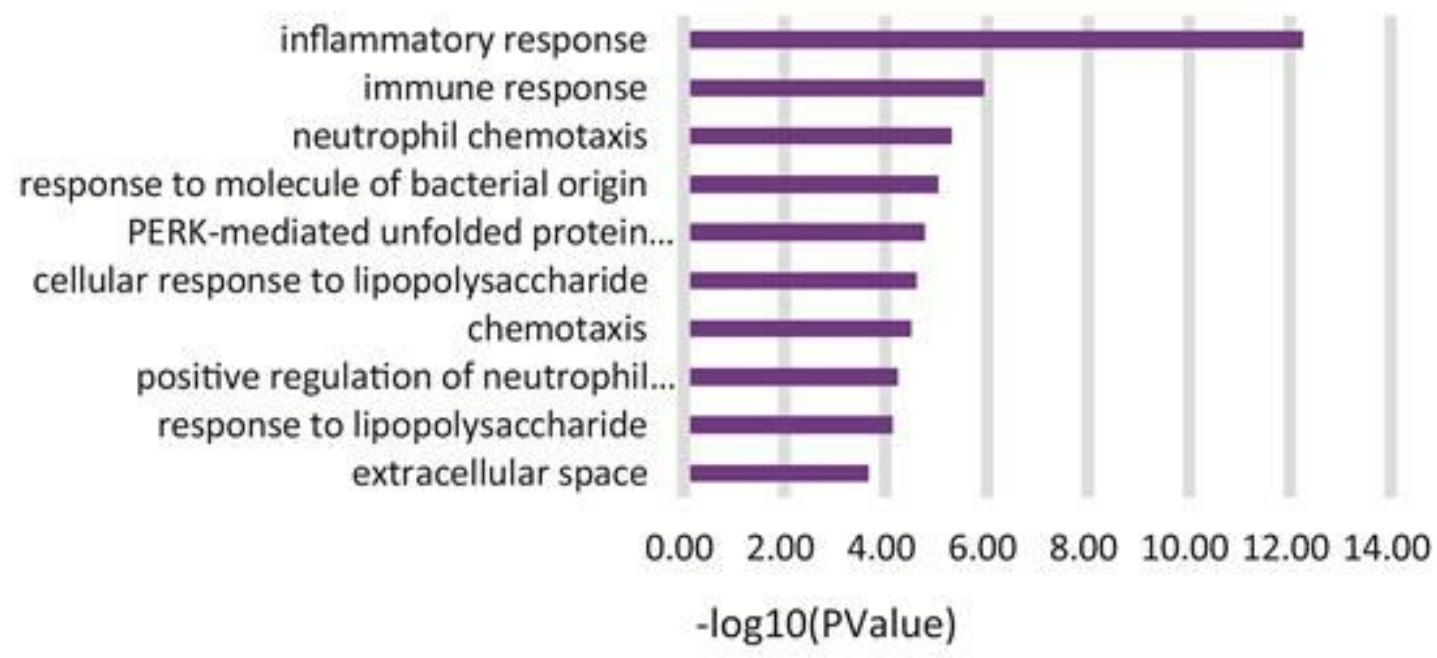

B

\section{The top 10 enriched KEGG pathway(cluster1)}

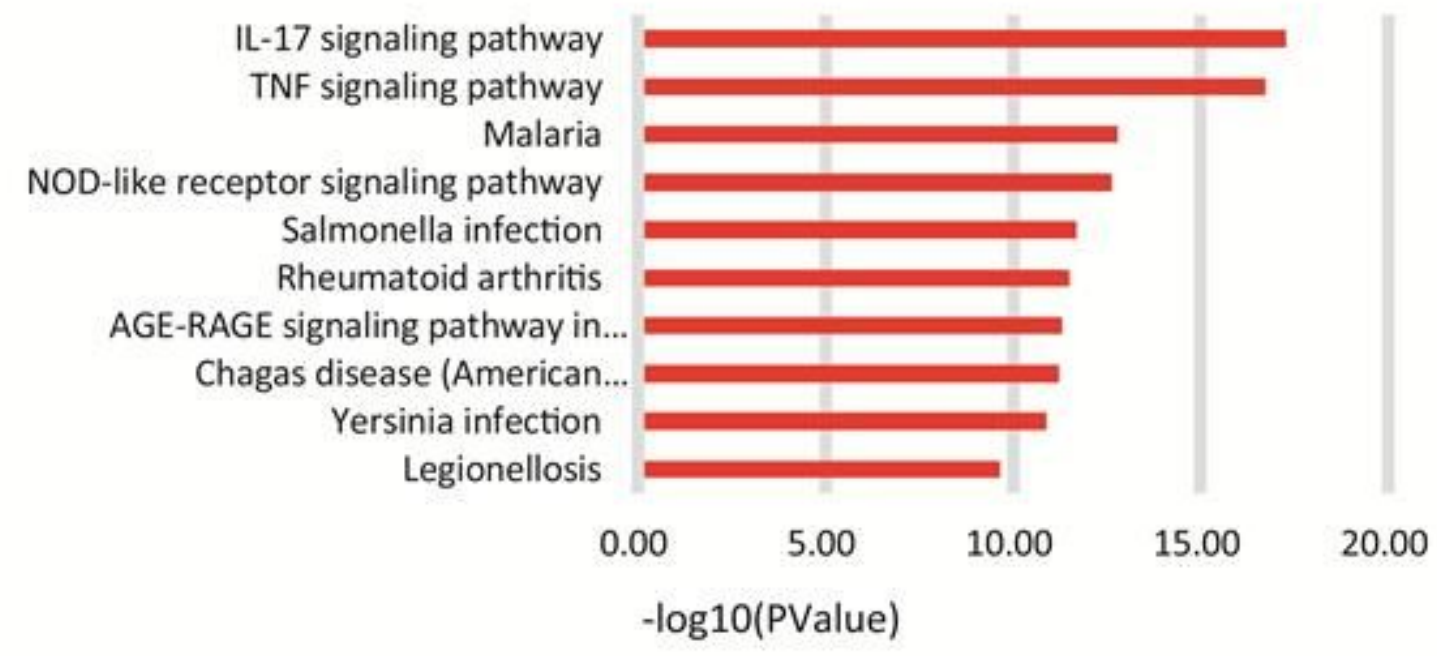

\section{Figure 5}

GO and KEGG enrichment analyses of cluster1; (A) The top 10 enrichen GO terms of cluster1; (B) The top 10 enrichen KEGG pathways of cluster1. 


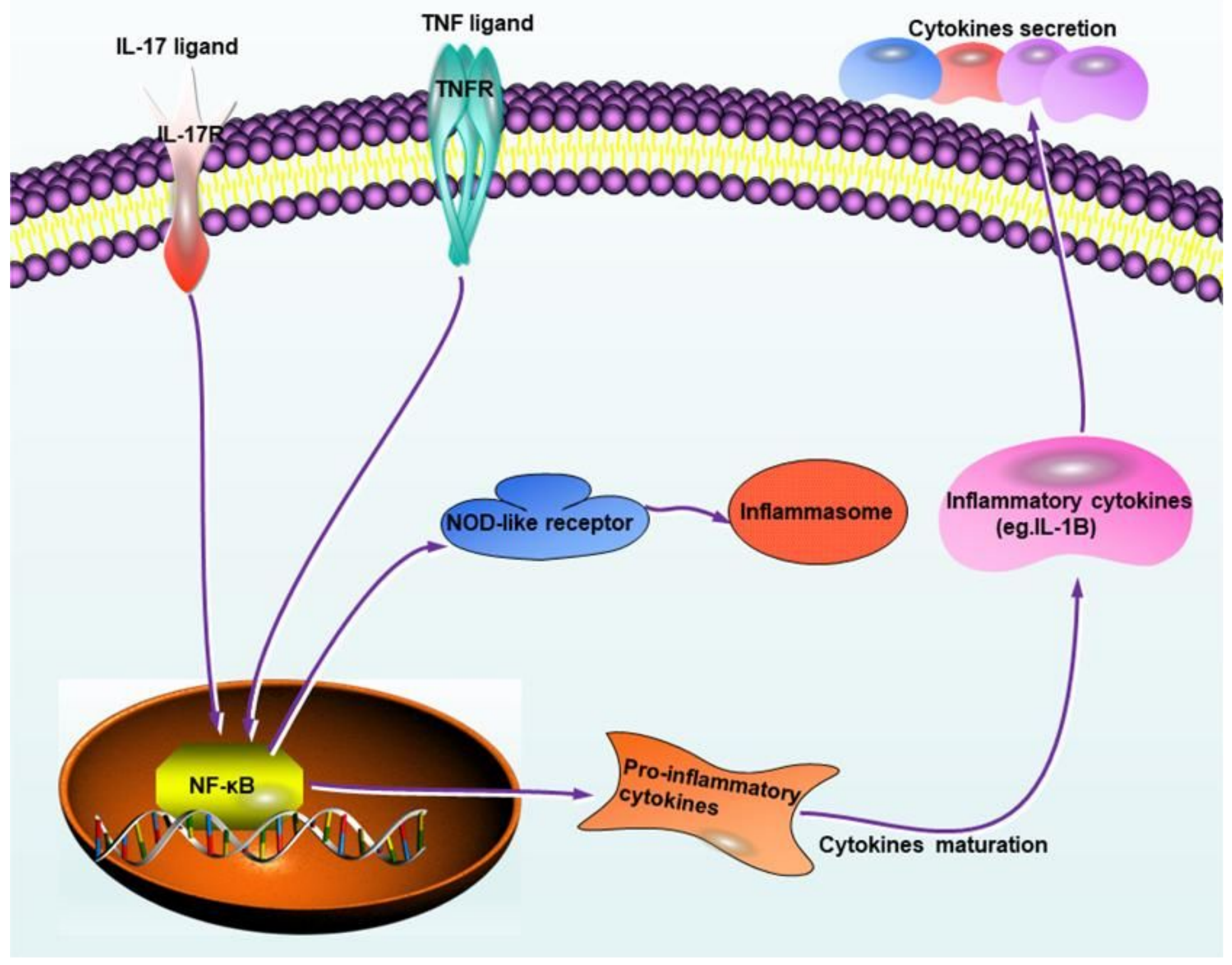

Figure 6

Hub genes were activated through IL-17 signaling pathway, TNF signaling pathway and NOD-like receptor signaling pathway. 